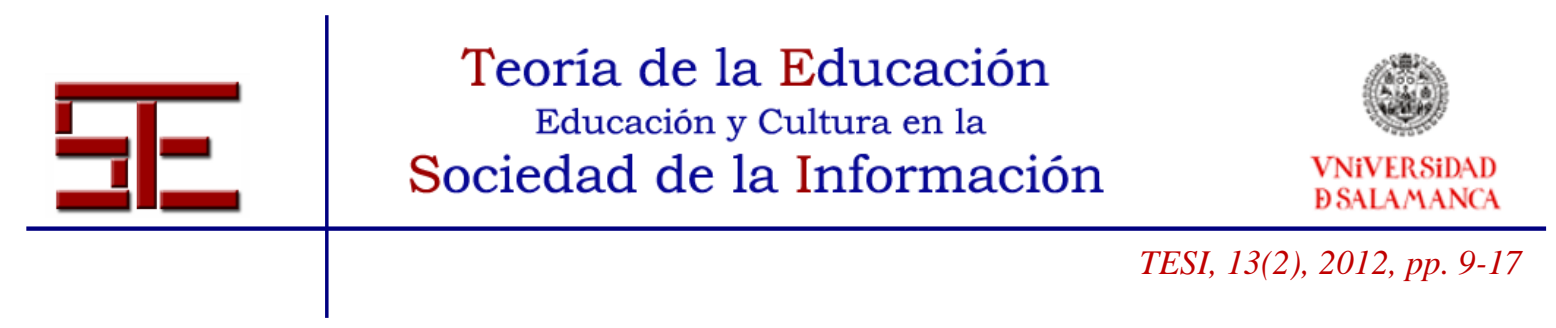

\title{
LA ROBÓtICA EDUCATIVA, UN NUEVO RETO PARA LA EDUCACIÓN PANAMEÑA.
}

Resumen: La robótica educativa es un nuevo reto para la educación panameña ya que además de abrir nuevos caminos dentro del currículo con la robótica en las aulas exige nuevos retos tanto para los docentes como los estudiantes.

Palabras clave: Robótica; Educativa; Kits

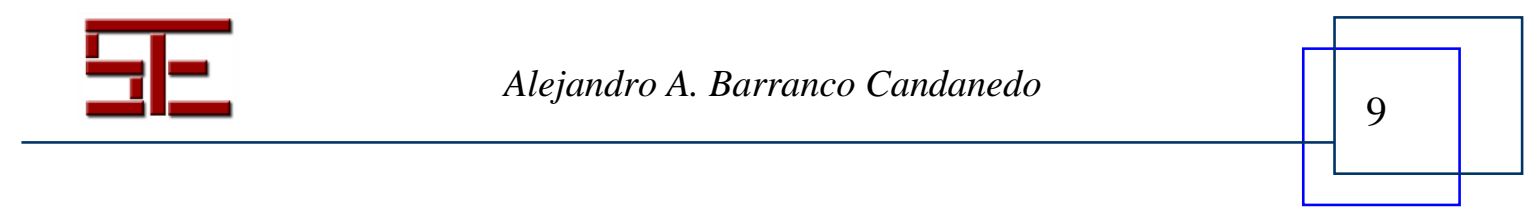


EDUCATIONAL ROBOTICS. A NEW CHALLENGE FOR THE PANAMANIAN EDUCATION

Abstract: The educational robotics a new challenge for Panamanian education break new ground in the school curriculum, this leads to introduce robotic technology in the classroom, which brings new challenges for teachers and students, who face high expectations.

Keywords: Robotics; Education; Kits

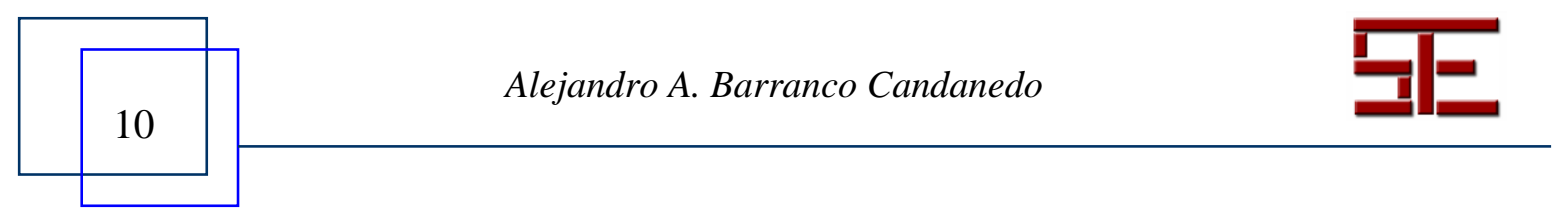




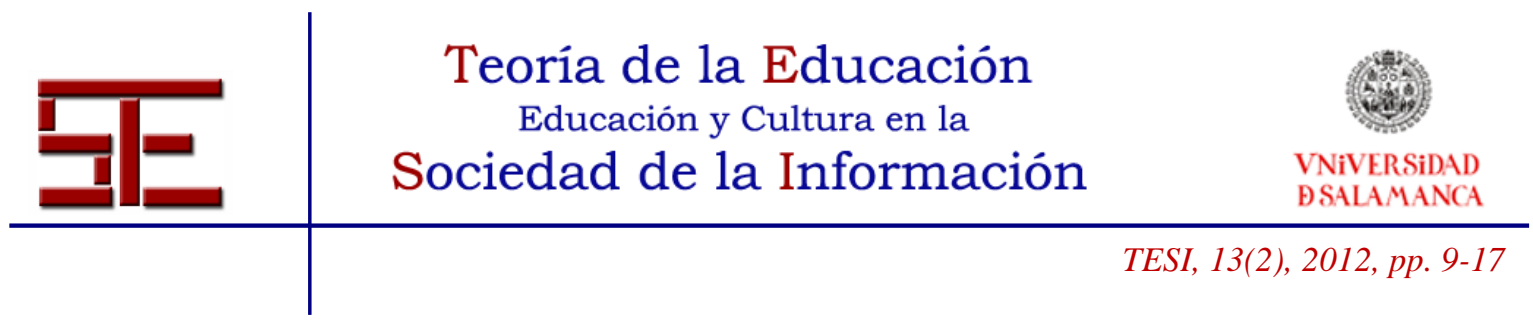

\title{
LA ROBÓTICA EDUCATIVA, UN NUEVO RETO PARA LA EDUCACIÓN PANAMEÑA
}

Fecha de recepción: 30/05/2012; fecha de aceptación: 14/06/2012; fecha de publicación: 26/07/2012

\author{
Alejandro A. Barranco Candanedo \\ aabarrancoc@yahoo.com
}

IPT Arnulfo Arias Madrid

\section{1.- LA ROBÓTICA EDUCATIVA EN PANAMÁ}

Hace algunos años, como pequeñas islas, los docentes fanáticos a los robots incursionamos hacia el mundo de la robótica educativa con situaciones de aprendizaje en las que participaban estudiantes. Ahora, cada vez que nos ofrecen la oportunidad de presentar nuestros avances tecnológicos en diferentes ferias escolares nos percatamos de que somos un continente de docentes y jóvenes que nos interesa esta área del conocimiento.

El concepto de Robótica Educativa lo enfoca claramente Acuña Zúñiga (2009: 2) cuando menciona que "concebimos la robótica educativa como un contexto de aprendizaje que se apoya en las tecnologías digitales para hacer robótica e involucra a quienes participan, en el diseño y construcción de creaciones propias, primero mentales y luego físicas, construidas con diferentes materiales y controladas por un computador llamadas simulaciones o prototipos". en donde nuestros estudiantes participan activamente en el desarrollo de estas actividades.

La visión del Ministerio de Educación de la República de Panamá $(2012 ; 24)$ de que "La robótica es vista en la sociedad actual como el área que mayor aplicación en la solución de problemas en el complejo ambiente informático y de la inteligencia artificial" advierte el documento que: "Panamá es el país más globalizado de la región; esto conlleva, además de una oportunidad económica y de compromiso social, la responsabilidad de estar preparados para atender una demanda significativa de profesionales de distintos sectores, en donde la aplicación de la tecnología a diversos procesos es requisito fundamental". Son argumentos que las autoridades panameñas y sus curriculistas consideraron para incorporar la robótica educativa como una asignatura más, dentro del pénsum académico para el duodécimo grado de la Educación Básica

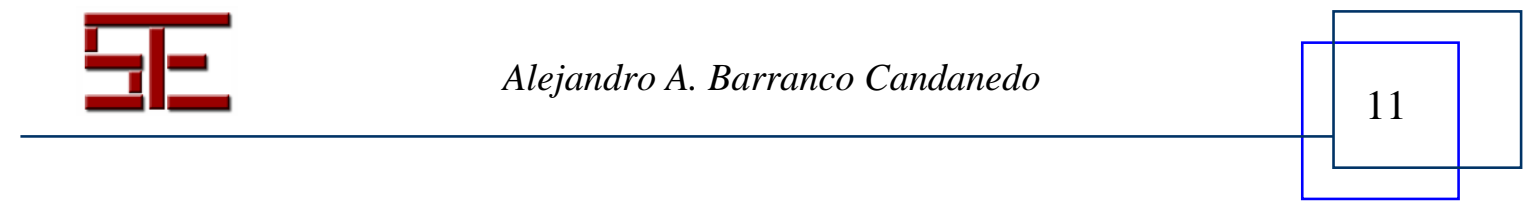




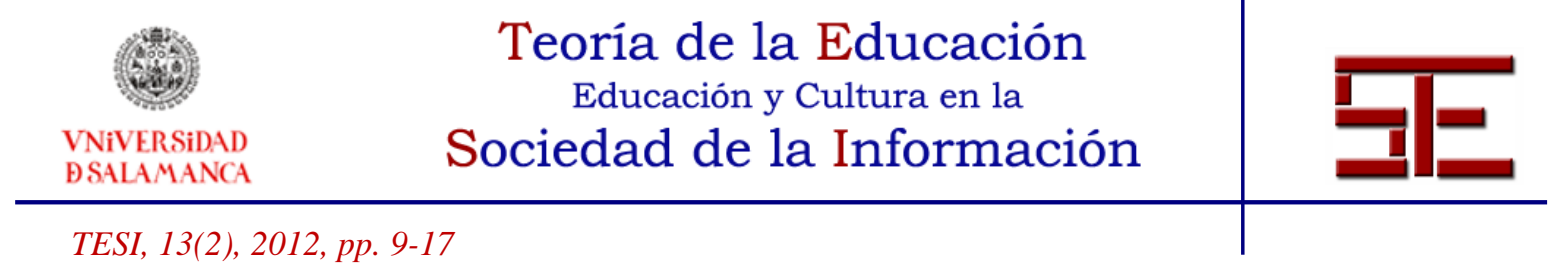

General, ya que hasta la fecha solo se realizaban actividades extracurriculares con docentes y estudiantes que tenían fascinación por el mundo de los robots.

En el 2011 se inició la Transformación Curricular en Panamá y con esta política educativa la apertura de un espacio para que los docentes interesados en la robótica introduzcamos a los participantes en esta tecnología, que les permite la creación de nuevos espacios de aprendizajes y ayuden a construir en nuestros estudiantes conocimientos de diversos tipos.

El Ministerio de Educación propone un nuevo reto a todos los docentes de tecnología informática que consiste en construir y programar robots sin tener experiencia previa, lo que obliga a los profesores a capacitarse de manera intensiva e inmediata, ya que son pocos los docentes a nivel nacional que cuentan con experiencia en robótica educativa y con poco o ningún conocimiento en la utilización de kits de robótica, que es fundamental para el buen desarrollo de la asignatura.

Esto pudiera considerarse una limitación que la mayoría de los docentes la ponderamos como buena: nos actualizamos por un lado y enseñamos lo aprendido a nuestros estudiantes por el otro. La expectativa que nos hemos propuesto es que los estudiantes puedan aprender de manera significativa conceptos que pudieran ser difíciles de entender y que de manera natural se apliquen y se ejecuten en las diferentes áreas del conocimiento con la ayuda de un kits de robótica como herramienta para resolver problemas del entorno donde ellos habiten; como también, el kit sea una instrumento que nos ayude a superar el reto principal.

\section{RETOS POR ENFRENTAR CON LA IMPLEMENTACIÓN DE LA ROBÓTICA EDUCATIVA}

Los retos a los que nos enfrentamos docentes y alumnos con la introducción de los Talleres de Robótica en el currículo escolar son muchos; sabemos que hay grandes expectativas, y más, cuando se desea realizar un cambio en los paradigmas educativos que tenemos.

El primer reto es quitarles el miedo a los docentes por el uso de la tecnología; como los estudiantes son nativos y nacen con el chip tecnológico preparado no habrá problemas. Una gran cantidad de docentes por primera vez observará y utilizará estos kits de robótica, y el poderles desaparecer ese miedo será fundamental para el buen desarrollo de sus habilidades en la construcción de robots.

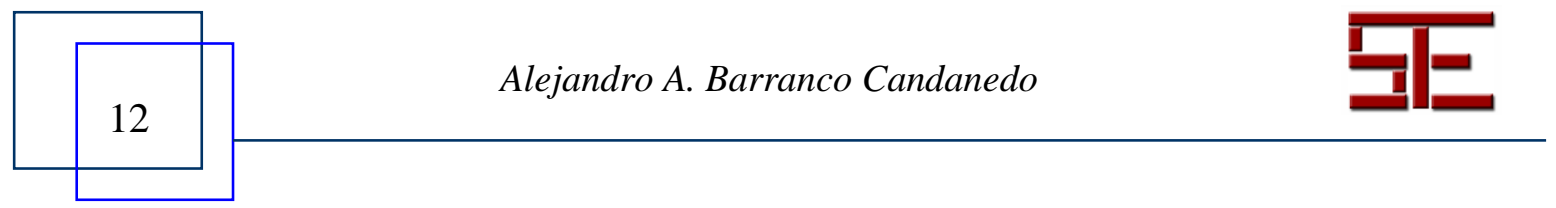




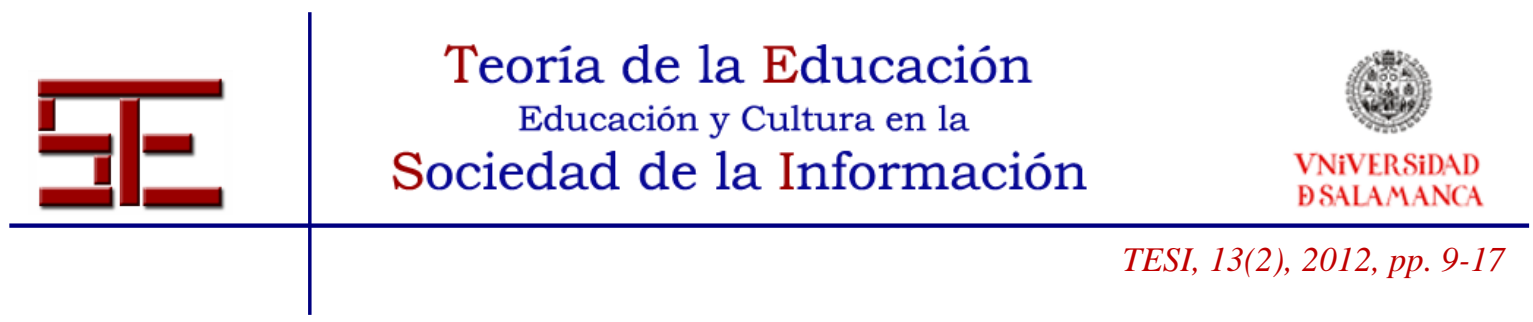

El segundo reto al que se enfrentarán los docentes será la falta de contacto con los kits de robótica; ya que, en la actualidad, son pocas las Escuelas Secundarias que tienen los kits como también, son pocas las que tienen la infraestructura para desarrollar los talleres y prácticas de robótica, limitante que obstaculiza una experiencia previa.

El tercer reto es la falta de bibliografía especializada en las bibliotecas de los colegios y el acceso a información de robótica en Internet resultará un desafío abrumador; ya que, por un lado, la gran cantidad de información multimedia sobre el tema saturaría al docente y al estudiante lo que no permitiría la fijación en el desarrollo de su problema para la adquisición de los conocimientos y experiencias significativas en la construcción de robots.

Por otro lado, el cuarto reto sería la necesidad de crear robots propios y guías de aprendizaje para que las experiencias en automatización sean panameñas y no extranjeras; de tal manera que el estudiante se enfoque la realidad donde convive diariamente.

La integración de otras áreas del conocimiento a la robótica a través de la revisión de los ejes transversales de las otras asignaturas que no son del área tecnológica como: español, física, matemática, entre otras; sería el quinto reto que muchos no le tomarán importancia, pero cuando la automatización se relacione con otras materias, este aprendizaje será fluido y enriquecedor; ya que los estudiantes lo harán de una manera natural y confortable; puesto que ya conoce cómo se construye un robot y aplicarán esos conocimientos en resolver los problemas en que se les asignen en otras asignaturas.

El Ministerio de Educación también tendrá su mayor reto en la implementación, todavía hay muchos planteles que no tienen las infraestructuras preparadas para impartir estos talleres de robótica, no han brindado información de cómo estará distribuido el espacio físico, el tipo de mobiliario, ni equipo de computadoras que se utilizará para programar los robots no se define a quién comprar los kits de robótica; no se cuenta con bibliografía que pueda servir de apoyo para preparar las clases que se dictarán, todo esto debe motivar al Ministerio de Educación a tomar las medidas necesarias para solventar estos inconvenientes.

El mayor reto para los docentes será que los estudiantes adquieran los conocimientos y las destrezas necesarias para utilizar eficientemente y de una manera divertida los kits de robótica y que les saquen el máximo provecho y obtengan un aprendizaje

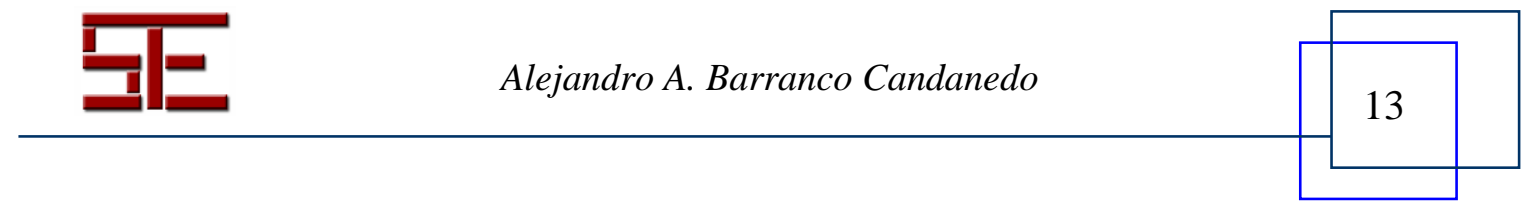




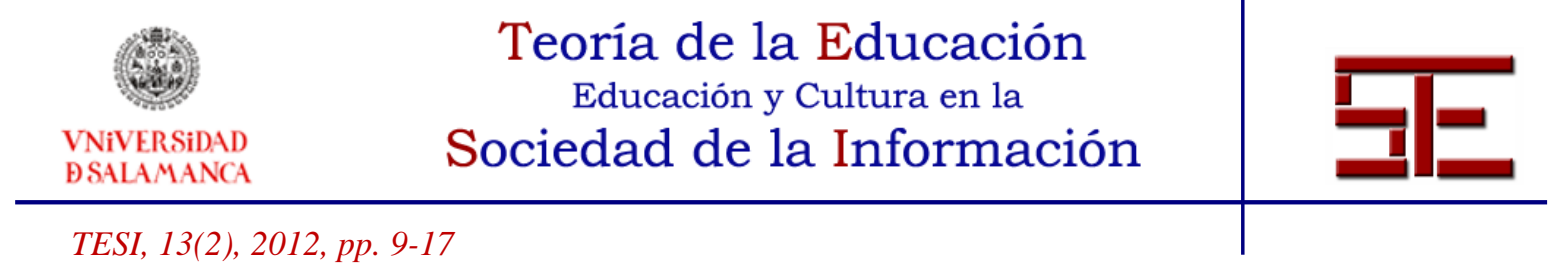

significativo; como menciona Ruiz-Velasco (2007: XV) "Se puede hacer un uso inteligente y racional de la tecnología, para generar entornos tecnológicos ricos, que permitan a los estudiantes la integración de distintas áreas del conocimiento para adquirir habilidades generales de información y comunicación y nociones científicas para la generación de conocimiento".

Los retos son muchos, pero lentamente se va viendo un rayo de luz al final del túnel; ya un alto porcentaje de docentes se capacitaron y están preparados para este nuevo desafío; actualmente se licita la construcción de los laboratorios y la adquisición de los kits de robótica, junto con la entrega de computadoras para los estudiantes, pero es imperativo que la capacitación a los docentes continúe, porque es necesario un contacto continuo con estos kits para que cada profesor pueda interrelacionar más con ellos y así buscar su propio aprendizaje para posteriormente brindarle las guías y ayuda pertinentes a los estudiantes. También es importante, tomar en cuenta la opinión de los docentes que impartirán estas asignaturas, ya que falta mucho camino por recorrer y hay que realizar ajustes en esta materia.

\section{EL PAPEL DEL DOCENTE EN LA ROBÓTICA EDUCATIVA}

Muchos docentes pensarán que estos kits de robótica son un juguete y que será fácil su construcción y utilización, pero se darán cuenta al primer contacto de que si no tienes experiencia con robótica, programación y diseño-construcción será una larga jornada de aprendizaje. A pesar de que el ambiente con los kits de robótica es muy amigable, se requiere de experiencia, conocimiento e interactividad con los kits, ya que con la práctica continua aprenderán y mejorarán las destrezas al utilizarlos.

El docente, parte esencial en este proceso, tendrá que jugar un papel primordial, como manifiesta Ruiz-Velasco (2007: 169) "El docente debe proveerse los medios de provocar las manifestaciones de conocimientos. Él tiene la necesidad de desarrollar una tipología de situaciones y de conocimientos. El saber se manifestará por las decisiones, la manera de hacer, las declaraciones y sus construcciones". Acuña Zúñiga (2004: 5) menciona que "Los profesores se proyectan como facilitadores de procesos de aprendizaje que permiten a los jóvenes asumir responsabilidades en un mundo cambiante. La mediación de los profesores tiene la intención de organizar los contextos y orientar los procesos de aprendizaje para favorecer la comprensión profunda de temas o problemas", estos son parte de los conceptos que se deben tener en cuenta

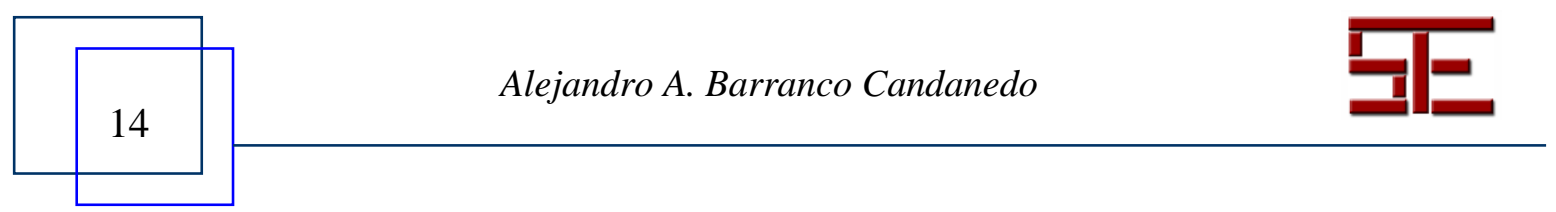




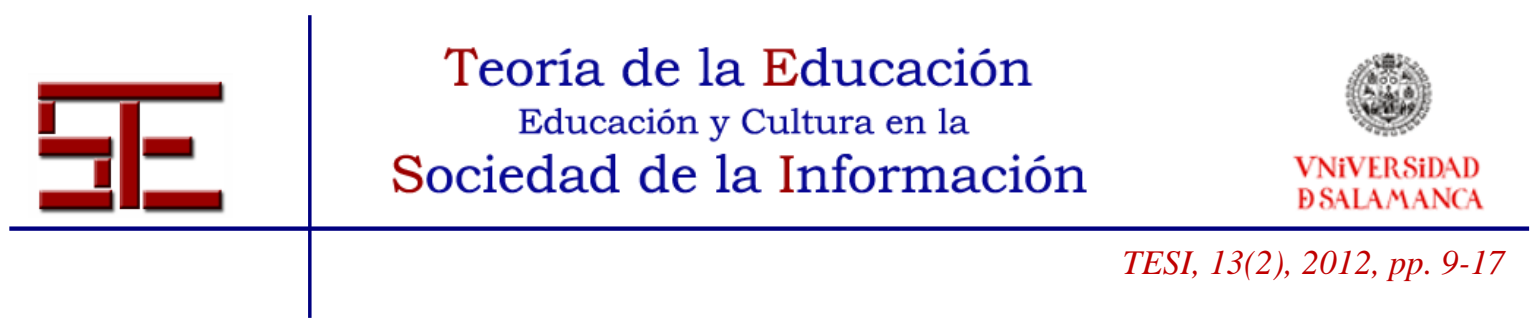

cuando se desea que el estudiante construya los robots, partiendo de sus conocimientos y del entorno de aprendizaje que cada uno desarrollara.

Las oportunidades que tienen los estudiantes en desarrollar su creatividad son enormes; en mi experiencia, he observado el entusiasmo y la creatividad que estos alumnos desarrollan, y se forma así no solo un aprendizaje técnico en los aspectos de diseño, construcción y programación, sino también, se notan cambios en su personalidad, como confianza en sí mismos, seguridad, liderazgo, autoestima; el buscar o crear desafíos y la habilidad para trabajar en equipo son algunas de las actitudes y aptitudes que tienen los estudiantes al relacionarse con la utilización de estos kits de robótica y con la ayuda del docente como facilitador de estas experiencias se pueden lograr.

La manera en que se introduzcan fácilmente conceptos de otras asignaturas será fundamental para la aplicación y ejecución de la robótica en otras materias, motivo por el cual, los docentes tendrán que formar alianzas con otros colegas para, así, llevar a una manera demostrativa algunos conceptos teóricos que se pueden resolver, utilizando un robot; es aquí, que el docente se involucrará en el proceso de enseñanza-aprendizaje de sus estudiantes como un ente motivador que lo induce a la investigación y promueve retos que puedan ser resueltos por estos.

El papel del docente en esta asignatura es fundamental ya que de la motivación, entusiasmo y dinamismo que el docente imponga, se reflejará en el trabajo de sus estudiantes y podrá recibir mejores resultados de aprendizaje. Este docente deberá promover la creatividad, el desarrollo de una mente imaginativa y sobre todo que el estudiante promueva su propio conocimiento y auto-aprendizaje, según Odorico (2004,: 34) "El profesor debe regular la actividad del alumno de forma adecuada respetando la actividad estructurante de éste. Su tarea consistirá en: detectar e interpretar los errores del alumno proponiendo alternativas para superarlos, proponer ayudas adecuadas al nivel de competencia del alumno, basar la ayuda en los conocimientos previos del alumno, proponer modelos de actuación que sirvan como ejemplos, sugerir nuevas metas y nuevas situaciones de resolución cuando decae el interés del alumno" asignándole problemas de diferentes grados de complejidad, promoviendo un pensamiento investigativo que lo lleve a buscar una solución a los problemas que se le presenten.

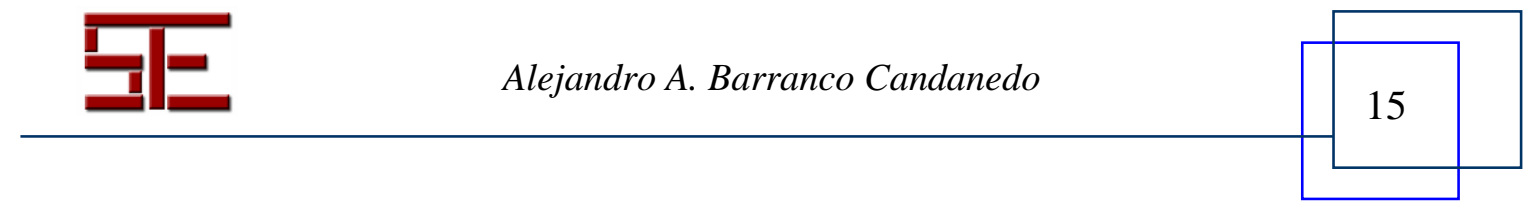




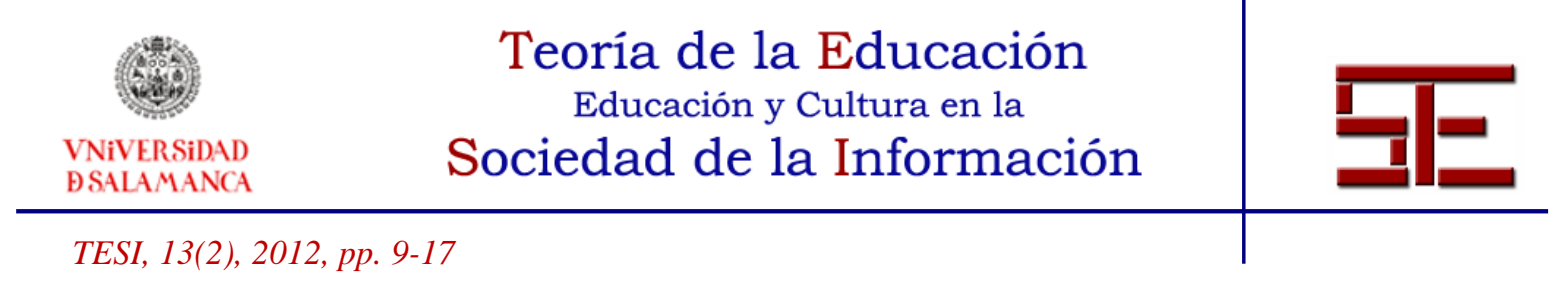

\section{CONCLUSIÓN}

La robótica educativa en Panamá tiene muchos retos, expectativas de qué pasará también las hay, al final la Robótica Educativa llegó para quedarse y promover en nuestros estudiantes la búsqueda del conocimiento y su propio auto-aprendizaje por medio de la utilización de kits y la integración de diferentes entes que componen el proceso de enseñanza-aprendizaje.

Dentro de la responsabilidad del Ministerio de Educación estará buscar la manera más eficiente para que los recursos de infraestructura, equipo, bibliografía y capacitación docente lleguen a los colegios a tiempo para el inicio del año escolar; de esta manera tener todo preparado para brindar un educación de calidad a nuestros estudiantes.

Los docentes tendrán un papel primordial, ya que, de sus habilidades, conocimientos y experiencias podrán enriquecer las clases y provocarán un ambiente de investigación, análisis y pensamiento crítico, promoviendo así un aprendizaje significativo.

También los docentes deberán actualizarse continuamente, promover la integración de diferentes asignaturas y propiciar la creación de bibliografía e investigación propia para generar aprendizajes y desarrollos en el entorno donde se desenvuelven los estudiantes, creando mejores condiciones para el aprendizaje.

Los estudiantes tendrán que involucrarse significativamente en su aprendizaje, ya que ellos tendrán que crear sus propios modelos, construir y programar los robots, validar sus resultados, cuestionar sus diseños e interactuar con otros compañeros y tomar las mejores decisiones para finalizar los desafíos que se les asignen.

Al final, los grandes beneficiarios de estos cambios en el currículo escolar serán los estudiantes, que podrán adquirir conocimientos y experiencias significativas, las cuales las enriquecerán como persona y desarrollarán como un ente productivo para la sociedad en la cual todos y cada uno de nosotros debemos poner nuestro grano de arena para solidificar la base de nuestra educación.

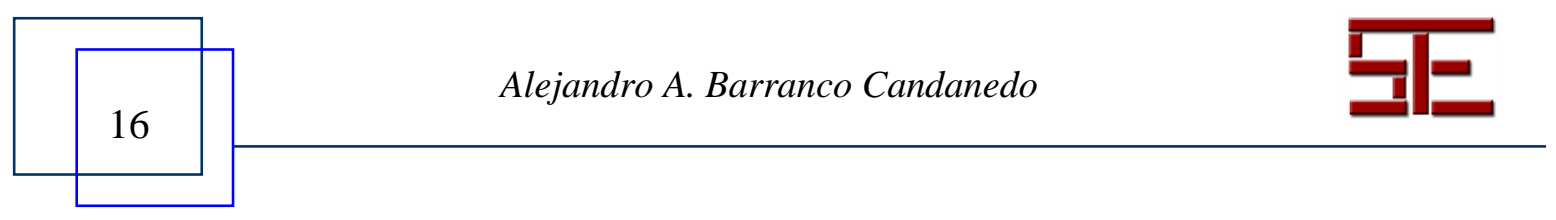




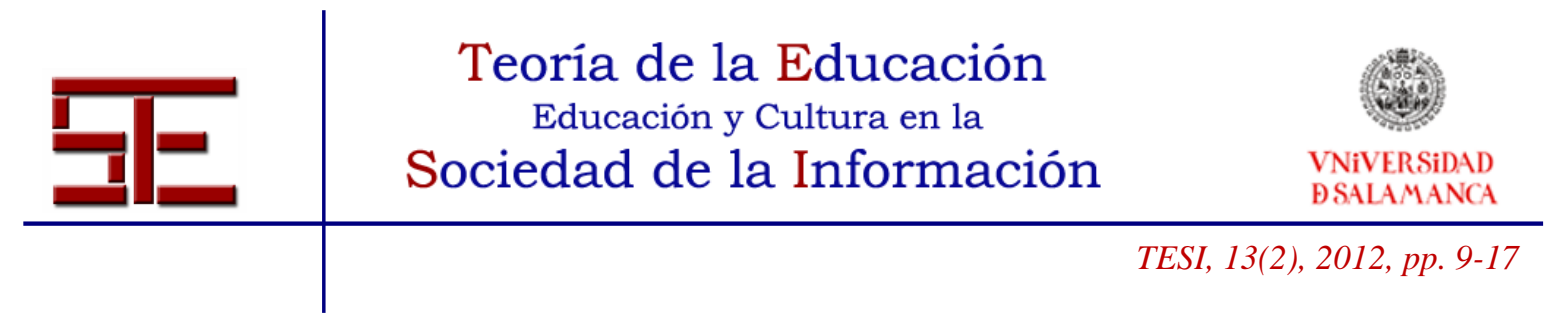

\section{BIBLIOGRAFÍA}

Acuña Zúñiga, A. L. (2004). Robótica y aprendizaje por diseño [en línea]. Extraído el 15 de mayo, 2012, de http://www.educoas.org/portal/bdigital/lae-ducacion/home.html. Organización de los Estados Unidos de America.

- (2009). La robótica educativa: un motor para la innovación. [en línea]. Extraído el 15 de mayo, 2012, de http://www.fod.ac.cr/robotica/descargas/roboteca/articulos/2009/motorinnova_ar ticulo.pdf.

Ministerio de Educación de Panamá (2012), Taller de Sistemas Robóticos, Programa de Educación Media, Duodécimo Grado. Panamá.

Odorico, A. (2004). Marco teórico para una robótica pedagógica [en línea]. Extraído el 13 de mayo, 2012, de http://laboratorios.fi.uba.ar/lie/Revista/Articulos/010103/A4oct2004.pdf.

Ruiz-Velasco Sánchez, E. (2007). Innovación en el aprendizaje de las ciencias y la tecnología. En su: Actuación del Alumno. España, Ediciones Díaz de Santos, pp 169.

Para citar el presente artículo puede utilizar la siguiente referencia:

Barranco Candanedo, A. A. (2012). La robótica educativa, un nuevo reto para la educación panameña. Revista Teoría de la Educación: Educación y Cultura en la Sociedad de la Información. 13(2), 9-17 [Fecha de consulta: dd/mm/aaaa]. http://campus.usal.es/ revistas_trabajo/index.php/revistatesi/article/view/8997/9242

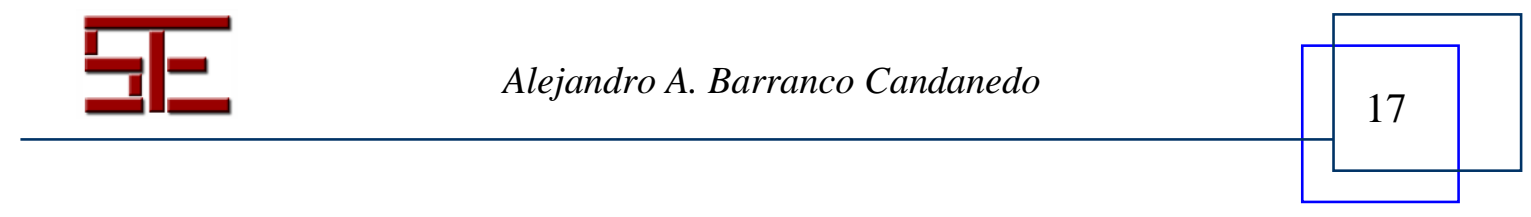

